\title{
Frequency shifting of pulsed narrow-band laser light in a multipass Raman cell
}

\author{
R. Sußmann, Th. Weber, E. Riedle ${ }^{1}$ and H.J. Neusser \\ Institut für Physikalische und Theoretische Chemie, Technische Universität München, \\ Lichtenbergstrasse 4,W-8046 Garching, Germany
}

Received 17 July 1991; revised manuscript received 1 October 1991

\begin{abstract}
A multipass cell is described which allows efficient stimulated Raman frequency shifting for low pump laser intensities and low gas pressures. The latter is important for Raman shifting of narrow-band Fourier-transform limited light pulses $(\Delta \nu=75 \mathrm{MHz})$. It is shown that frequency broadening of the Raman shifted light can be largely avoided in the Dicke narrowing regime at low pressures. For $75 \mathrm{MHz}$ pump pulses and an $\mathrm{H}_{2}$ density of 2.5 amagat we found a negligible broadening to $90 \mathrm{MHz}$ of the stimulated Stokes light. This is far below the value of $250 \mathrm{MHz}$ expected from spontaneous emission. The narrow-band Stokes pulses achieved in $\mathrm{CO}_{2}$ enabled us to measure the pressure shift coefficient $\left(-0.71 \times 10^{-2} \mathrm{~cm}^{-1} /\right.$ amagat $)$ of this gas. It is demonstrated, for the example of benzene, that our technique provides a very practical light source for high resolution molecular spectroscopy.
\end{abstract}

\section{Introduction}

The tuning range of narrow-band single-mode dye laser systems is limited by the wavelength range and efficiency of the dyes and the available pump laser frequencies. Therefore it is highly desirable to develop a technique for frequency shifting of narrow frequency light without loss of spectral selectivity. Stimulated Raman scattering (SRS) in high density gases is known to be a convenient technique for frequency converting tunable dye laser emission, but has so far been used only for broader bandwidths.

Until 1990, no successful attempt had been made to reduce the bandwidth of Raman shifted light to its physical limits $[1,2]$. For a pump laser with negligible linewidth, the bandwidth of the Raman shifted light is determined by pressure broadening in the high density regime of the gaseous Raman medium $\left(\mathrm{H}_{2}\right.$ : $\rho>5$ amagat ) and by Doppler broadening in the low density regime $\left(\mathrm{H}_{2}: \rho<2\right.$ amagat $)$.

Dicke narrowing [3] is expected to lead to a nar-

1 Present address: Joint Institute for Laboratory Astrophysics, University of Colorado and National Institute of Standards and Technology, Boulder, CO 80309-0440, USA (1991-1992 visiting fellow). rowing of the linewidth of the Raman transition below these limits in a specific density regime. This has been observed by stimulated Raman backward scattering in $\mathrm{H}_{2}$ [4], in spontaneous Raman forward scattering [5], and with the method of stimulated Raman gain spectroscopy [6-8]. In $\mathrm{H}_{2}$ a minimum spontaneous Stokes linewidth of $250 \mathrm{MHz}$ was observed at a density of 2.5 amagat. Another narrowing process is caused by the Q-branch collapse (line mixing) phenomenon [9]. In the Raman $\nu_{1}$ band of $\mathrm{CO}_{2}$, both narrowing processes occur in same density regime of about 0.3 amagat $[10,11]$.

Efficient Raman shifting of laser light in single-pass cells requires pressures of several bar [12]. Due to their increased interaction lengths, multi-pass cells are advantageous because they reduce the SRS threshold pump power [13] and allow the pressure to be reduced to the narrowing regime. With this technique and a low pressure of 900 Torr, Saint-Loup et al. achieved a linewidth of $120 \mathrm{MHz}$ (fwhm) at a Stokes conversion efficiency of about $12 \%$ in the Raman medium $\mathrm{CO}_{2}$ [2]. However, no data are available for the most common Raman medium $\mathrm{H}_{2}$.

In this work a new multipass cell that produces a narrowing beyond the spontaneous linewidth in $\mathrm{H}_{2}$ and beyond the $\nu_{1}$ bandwidth in $\mathrm{CO}_{2}[10,11]$ is de- 
scribed. For the first time a linewidth of the Raman shifted light of less than $100 \mathrm{MHz}$ is obtained in $\mathrm{H}_{2}$.

\section{Experimental apparatus}

Our experimental setup for narrow-band stimulated Raman shifting is shown in fig. 1. A multipass unit was designed and constructed for Stokes and anti-Stokes shifting of the extremely narrow-band light of a pulsed amplified $\mathrm{cw}$ single mode dye laser in the Raman media $\mathrm{H}_{2}$ (shift: $4155 \mathrm{~cm}^{-1}$ ) and $\mathrm{CO}_{2}$ (shift: $1388 \mathrm{~cm}^{-1}$ ). The setup consists of a modematching telescope [14], the multipass optics and the gas cell. A lens is used to receive a collinear beam at the exit of the optics, and a dispersion prism separates the fundamental and the frequency-shifted light.

For the multipass optics the Herriot design [15] was chosen. It combines a long optical path length with periodic refocusing and therefore provides reasonable conversion efficiency for the given pump pulse energy of 4-5 mJ and the low gas densities necessary for the narrowing. In previous works on Raman shifting with multipass optics the mirrors were positioned inside the gas cell containing the Raman medium $[2,13]$. In this work we decided to place the mirrors outside. This resulted in simple manufacturing and handling and enabled us to optimize the operation parameters of the cell easily.

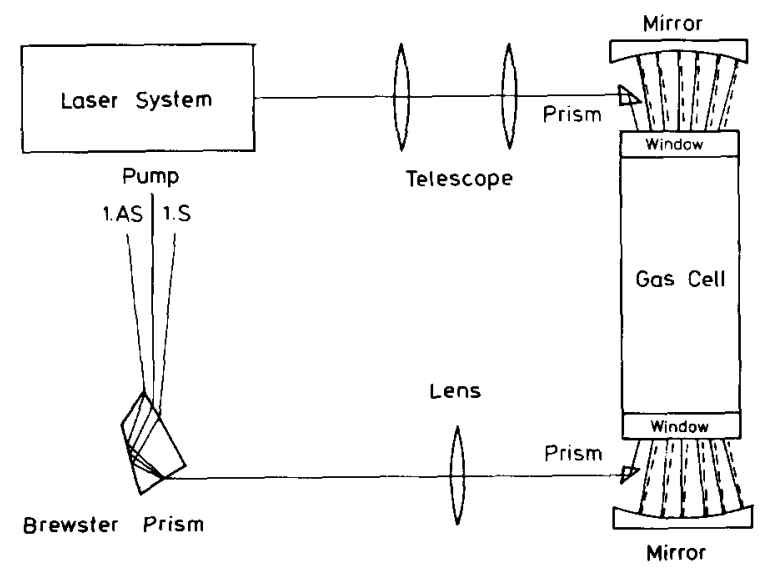

Fig. 1. Experimental setup for narrow-band Raman shifting. The mirrors of the multipass unit are placed outside the cell containing the gaseous Raman medium.
The inherent problems of this new design are additional losses and depolarization of the light at the windows of the intermediate gas cell [16]. In the small signal limit, the gain $G$ in the Stokes output power due to the multipass arrangement is only dependent on the losses and the number $n$ of passes through the gas cell $[2,13]$

$G \simeq\left(T_{\text {efrs }}\right)^{n-1} \frac{1-\left(T_{\text {eff }}\right)^{n}}{1-\left(T_{\text {eff P }}\right)}$,

with $T_{\text {efrS }, \mathrm{P}}=R_{\mathrm{S}, \mathrm{P}} T_{\mathrm{S}, \mathrm{P}}^{2}$. Here $R_{\mathrm{S}}$ and $R_{\mathrm{P}}$ are the mirror reflectivity and $T_{\mathrm{S}}$ and $T_{\mathrm{P}}$ the transmission of one cell window at the pump and Stokes wavelength, respectively. $T_{\text {effs }}$ and $T_{\text {eff } P}$ represent the effective transmission for a single pass. Highly reflecting mirrors $\left(R_{\mathrm{S}} \approx R_{\mathrm{P}} \geqslant 99.5 \%\right)$ and antireflex coated windows with $T_{\mathrm{S}} \approx T_{\mathrm{P}} \geqslant 99.3 \%$ yielded a multipass gain of $G=13$ for $n=26$ passes through the cell. Theoretically, a total cell transmittance of $60 \%$ is expected after 26 passes through the cell. The second inherent problem of our cell design is the strain birefringence of the pressure vessel windows. This causes a depolarization of the light, which strongly decreases the efficiency of frequency doubling necessary for an application in uv spectroscopy. The strain was controlled and minimized in an optical strain analyzing device.

Mode matching [14] fits the curvature of the wavefronts of the beam to that of the mirrors. Thus the spot radius on the mirrors (hwhm) $w$ and the beam waist $w_{0}$ follow the equations [17]

$w_{0}^{2}=\frac{L \lambda}{\pi} \sqrt{\frac{1+g}{4(1-g)}}, \quad w^{2}=\frac{L \lambda}{\pi} \sqrt{\frac{1}{1-g^{2}}}$,

with $g \equiv 1-L / R$. $L$ is the distance between the mirrors of curvature $R$ and $\lambda$ the pump wavelength. Real and finite solutions for the spot radius $w$ exist only if $0 \leqslant g^{2} \leqslant 1$. From eq. ( 2 ) it can be seen that a nearly concentric geometry $(L / R \lesssim 2)$ provides tightest focusing (thereby enhancing the Raman gain [13]). From eq. (2) it is also recognized that maximum $w$ is obtained for $(L / R \lesssim 2)$ and large cell dimensions $(R, L)$, thus reducing the light intensity at the mirrors. Thus mirrors with $R=500 \mathrm{~mm}(L \approx 950 \mathrm{~mm})$ were chosen, leading to a reasonable and convenient experimental setup and operating parameters well below mirror and cell window damage thresholds. 
The beam of the pump laser is injected from one side of the multipass cell using a small adjustable right angle prism and leaves the cell on the other side by means of an identical prism (fig. 1). The light spots on the mirrors resulting from the different passes through the cell are located on a circle. Thus the spacing $d$ between two neighboring spots must be larger than the size of the prism $(d>3 \mathrm{~mm})$. Mirror substrates with $60 \mathrm{~mm}$ diameter are most suitable for these conditions, since $n=36$ is the number of passes that provides optimum gain in the small gain limit (eq. (1)) and thus an upper limit for efficient conversion in the high gain regime.

The gas cell was constructed to withstand pressures up to $7 \mathrm{~atm}$ with fused silica windows of 10 $\mathrm{mm}$ thickness $(\varnothing=50 \mathrm{~mm})$. The cell length of 600 $\mathrm{mm}$ was chosen to be larger than twice the Rayleigh range $\left(z_{\mathrm{R}}=100 \mathrm{~mm}\right)[17]$, where most of the conversion occurs.

The output of a single-mode cw dye laser (CR 699) was amplified in a three-stage amplifier to produce the narrow-band tunable pulsed laser light in the wavelength range $470-520 \mathrm{~nm}$. The three amplifiers were pumped by the output of a $\mathrm{XeCl}$ excimer laser (EMG 150). At a wavelength of about $510 \mathrm{~nm}$, light pulses of $5 \mathrm{~mJ}$ energy and $10 \mathrm{~ns}$ duration (fwhm) are produced from pump pulses of $100 \mathrm{~mJ}$. Their frequency width is about $75 \mathrm{MHz}$, and thus nearly Fourier-transform limited $[18,19]$.

\section{Experimental results and discussion}

\subsection{General operation conditions}

In fig. 2a the dependence of the Stokes pulse energy $E_{\mathrm{S}}$, measured for three different numbers of passes in $\mathrm{CO}_{2}$, is plotted as a function of the gas pressure. It may be seen that for a constant pump pulse energy $\left(E_{\mathrm{P}}=4.4 \mathrm{~mJ}\right)$ the pressure needed to achieve a reasonable conversion efficiency can be significantly reduced for an increasing number of passes. The dashed horizontal line indicates the $7.5 \%$ conversion efficiency. For a pressure of 5 bar this conversion efficiency was obtained with 8 passes, for a lower pressure of 2 bar with 12 passes, and for the lowest pressure of 1.2 bar with 24 passes. It should be noted that the distance $L$ between the mirrors has to be readjusted for each number of passes [2].

In fig. $2 b$ the pulse energy of the first Stokes emission, $E_{\mathrm{S}}$, is plotted as a function of the pump pulse energy, $E_{\mathrm{P}}$, at a pressure of $p=3$ bar. Here it is recognized again that the required pump pulse energy can be reduced when the number of passes through

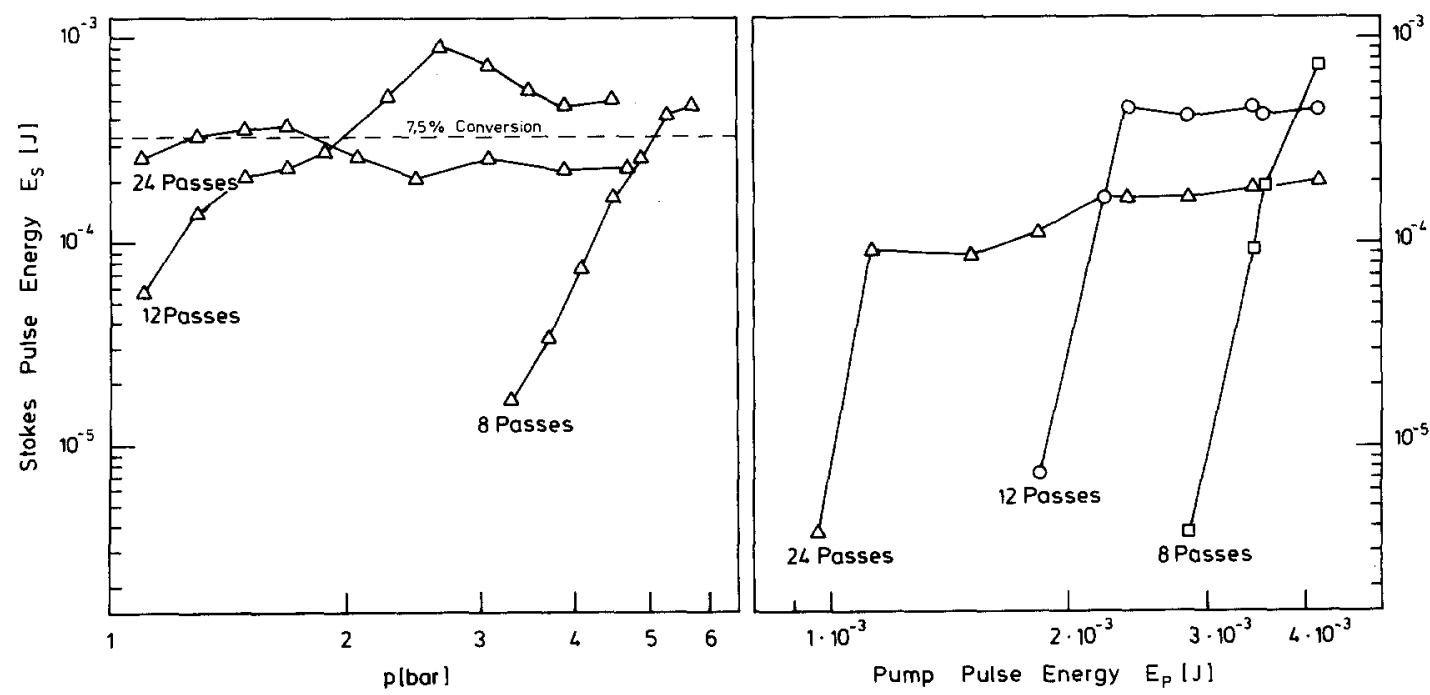

Fig. 2. Pulse energies $E_{\mathrm{R}}$ of first Stokes emission in $\mathrm{CO}_{2}$ for various numbers of passes in the multipass cell as a function, (a) of the gas pressure $p$ (constant pump pulse energy $E_{\mathrm{P}}=4.4 \mathrm{~mJ}$ ) and (b) of the pump pulse energy $E_{\mathrm{P}}$ (constant pressure $p=3 \mathrm{bar}$ ). 
Table 1

Typical data of Stokes (S) and anti-Stokes (AS) shifting in $\mathrm{CO}_{2}$ and $\mathrm{H}_{2}$ with the multipass cell at low pressures: Raman pulse energy $\left(E_{\mathrm{R}}\right)$, pump pulse energy $\left(E_{\mathrm{P}}\right)$, conversion efficiency $\left(E_{\mathrm{R}} / \dot{E}_{\mathrm{P}}\right)$, wavelength of pump light $\left(\lambda_{\mathrm{P}}\right)$, wavelength of the Raman shifted light $\left(\lambda_{\mathrm{R}}\right)$ and its minimum linewidth $\left(\Delta \nu_{\min }, \mathrm{fwhm}\right)$. The pump laser linewidth is $75 \mathrm{MHz}$ (fwhm).

\begin{tabular}{llcccccc}
\hline & & $\begin{array}{l}E_{\mathrm{R}} \\
(\mu \mathrm{J})\end{array}$ & $\begin{array}{l}E_{\mathrm{R}} / E_{\mathrm{P}} \\
(\%)\end{array}$ & $\begin{array}{l}\Delta \nu_{\min } \\
(\mathrm{MHz})\end{array}$ & $\begin{array}{l}\lambda_{\mathrm{R}} \\
(\mathrm{nm})\end{array}$ & $\begin{array}{l}\lambda_{\mathrm{P}} \\
(\mathrm{nm})\end{array}$ & $\begin{array}{l}\text { Number of } \\
\text { passes }\end{array}$ \\
\hline $\mathrm{H}_{2}$ & 1st S & 1000 & 27.2 & $90(6)$ & 647 & 485 & 12 \\
3.5 amagat & 1st AS & 38 & 1.0 & $100(20)$ & 421 & 485 & 12 \\
$\mathrm{CO}_{2}$ & 1st S & 455 & 16.0 & $117(21)$ & 520 & 485 & 24 \\
0.9 amagat & 2nd S & 418 & 12.5 & $220(21)$ & 594 & 510 & 24 \\
& 3rd S & 327 & 9.8 & - & 647 & 510 & 26 \\
& 1st AS & 64 & 2.8 & $146(23)$ & 454 & 485 & 26 \\
& 2nd AS & 12 & 0.4 & - & 447 & 510 & 28 \\
\hline
\end{tabular}

the cell is increased. This means conversely that for a defined pump pulse energy the gain increases when the number of passes is increased. It will be shown below that this high gain is responsible for additional narrowing of the Stokes linewidth.

In order to achieve maximum conversion efficiency (table 1) of the Stokes and anti-Stokes shifted components of different orders, the number of passes had to be adjusted separately in each case. Typically, for $\mathrm{CO}_{2}$ at the required low density of 0.9 amagat and a pump pulse energy of $3 \mathrm{~mJ}$, conversion efficiencies of $16 \%$ for the first Stokes emission ( 24 passes) and $2.8 \%$ for the first anti-Stokes emission (26 passes) were achieved. Similarly, for an $\mathrm{H}_{2}$ density of 3.5 amagat a high Stokes conversion efficiency of $27 \%$ and an anti-Stokes conversion efficiency of $1 \%$ were achieved in 12 passes.

\subsection{Linewidth}

The linewidth of the Raman $Q_{01}(1)$ transition of $\mathrm{H}_{2}$ in the Dicke narrowing regime has been investigated by spontaneous measurements [5] and by stimulated Raman gain spectroscopy [6-8]. The results [8] are in line with the relation

$\Delta \nu_{\text {Dicke }}=\frac{D_{0} k^{2}}{\pi \rho}+a \rho$.

Here $D_{0}\left(1.39 \mathrm{~cm}^{2}\right.$ amagat/s $)$ is the self-diffusion constant, $k$ the difference vector between the wave vectors of the incident laser and emitted Stokes photons, and $a$ ( $51.3 \mathrm{MHz} /$ amagat) is the pressurebroadening coefficient. This relation is represented by the solid line in the upper part of fig. 3a. It displays a minimum of the Raman linewidth of 250 $\mathrm{MHz}$ at a density of 2.5 amagat.

For comparison, in the lower part of the figure, the experimental results of this work are shown. They have been achieved by stimulated Stokes shifting in the multipass cell. The linewidth of both the pump and the Stokes light was measured with a high resolution confocal Fabry-Perot interferometer. Care was taken to maximize the pulse length of the Stokes light as to minimize its linewidth. Comparing the new results with the curve in the upper part of the figure reveals three striking differences.

(i) The minimum linewidth of $90 \mathrm{MHz}$ achieved in SRS is significantly lower than the value of 250 $\mathrm{MHz}$ of the $\mathrm{Q}_{01}(1)$ transition [8] and is close to the exciting laser linewidth of $75 \mathrm{MHz}$; substraction of the laser linewidth (assuming for simplification lorentzian lines) would result in a linewidth of $15 \mathrm{mHz}$ for the broadening caused by SRS in the multipass cell.

(ii) The linewidth minimum is shifted to higher densities (3.5-4 amagat).

(iii) The measured increase of the linewidth at higher densities (due to pressure broadening) is less than expected from spontaneous Raman data [5] and from stimulated Raman gain spectroscopy [8].

The effects described above for $\mathrm{H}_{2}$ are also found for the Stokes-shifted SRS emission in $\mathrm{CO}_{2}$ gas (fig. $3 \mathrm{~b}$, lower part). For comparison, the density dependence of the $\nu_{1}$ full bandwidth of $\mathrm{CO}_{2}$ measured by stimulated Raman gain spectroscopy [11] is shown in the upper part. Recently, the additional narrowing 

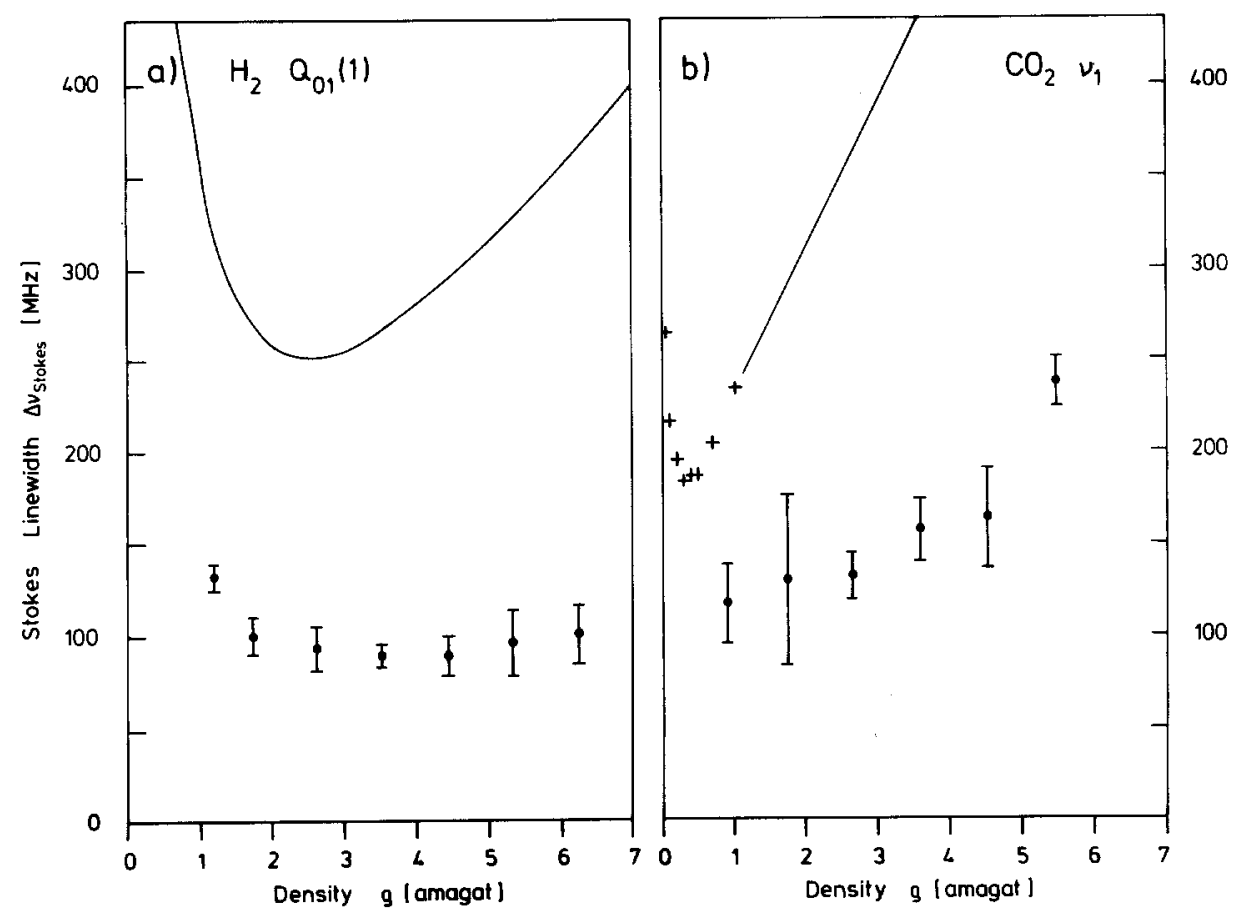

Fig. 3. (a) Upper part: theoretical dependence of the linewidth of the Raman $Q_{01}(1)$ transition in $H_{2}$ (eq. (3)) in good agreement with measurements of ref. [8]. Lower part: measured first Stokes linewidths for different gas densities in the multipass cell (this work). The error bars represent the accuracy of the frequency measurements and three standard deviations of the average value of several measurements. (b) Upper part: density dependence of the $\nu_{1}$ full bandwidth of $\mathrm{CO}_{2}$ measured by stimulated Raman gain spectroscopy ( + ) [11]; the solid line represents the linear pressure broadening. Lower part: measured first Stokes linewidths for different gas densities in the multipass cell (this work).

(i) of SRS was observed in $\mathrm{CO}_{2}$ at a density of 1.2 amagat $[20]$.

The effects (i)-(iii) are caused by the nature of the high gain stimulated Raman effect in the multipass cell leading to the well known gain narrowing phenomenon. Assuming a simple amplification model [17] the linewidths of the spontaneous and stimulated signals (assuming for simplification lorentzian lines) are related by

$\Delta \nu_{\mathrm{SRS}}=\Delta \nu_{\mathrm{spEm}} \sqrt{\frac{\ln 2}{\ln \left(G_{\mathrm{SRS}} / 2\right)}}$,

where $G_{\mathrm{SRS}}$ is the total Raman gain. For $G_{\mathrm{SRS}} \sim \mathrm{e}^{30}$ and $\Delta \nu_{\mathrm{sp} E \mathrm{~m}}=250 \mathrm{MHz}$ the minimum linewidth for $\mathrm{H}_{2}$ eq. (4) yields $\Delta \nu_{\mathrm{SRs}} \approx 38 \mathrm{MHz}$. This is in reasonable agreement with the value of $15 \mathrm{MHz}$ in our experiment. For broader laser linewidths and higher densities stronger narrowing than predicted by eq.
(4) has been observed in previous work [6,21]. With eq. (4) the results (ii) and (iii) can be explained by the higher gain expected for higher densities. Here gain narrowing becomes even more evident.

From figs. $3 a$ and $3 b$ it is seen that narrow-band Stokes-Raman shifting is possible at higher densities than predicted by spontaneous linewidth measurements $\left(\mathrm{H}_{2}[5]\right)$ and stimulated Raman gain spectroscopy $\left(\mathrm{H}_{2}[8], \mathrm{CO}_{2}[10,11]\right)$; similar results were found for anti-Stokes shifting (table 1). This is of great practical importance as it leads to a high conversion efficiency for the Raman shifted light.

\subsection{Pressure shift}

For the narrow-band Raman shifted light of the present work $(\Delta \nu=90-120 \mathrm{MHz}$, see table 1) the pressure shift in the Raman medium has to be considered. Using the multipass cell, the variation of the 


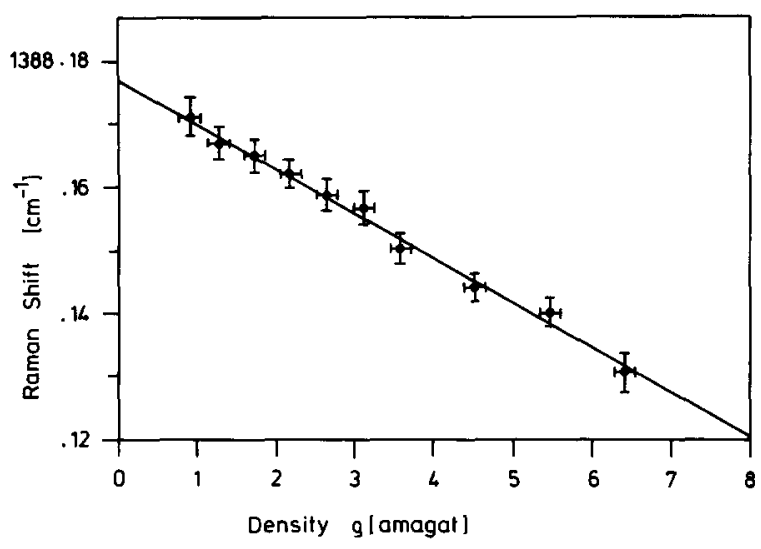

Fig. 4. Measured pressure shift of the Raman Stokes light of $\mathrm{CO}_{2}$. The solid line represents a linear least-squares fit to the experimental points, see eq. (5).

Raman frequency shift of the collapsed $\nu_{1}$ Q-branch $[10,11]$ as a function of the gas density was accurately determined for $\mathrm{CO}_{2}$ gas, as shown in fig. 4. For absolute frequency calibration, the iodine absorption spectra were recorded [22] simultaneously, both in the frequency range of the fundamental laser and the Stokes pulses. The measured Raman shift $\nu$ displays a linear dependence on the gas pressure and was found to follow the equation (solid line in fig. 4)

$$
\nu=1388.177(5)-0.71(10) \times 10^{-2} \rho .
$$

Here $\nu$ is the Raman shift in $\mathrm{cm}^{-1}$ and $\rho$ the density in amagat.

\section{Application to high resolution molecular uv spectroscopy}

The Raman Stokes light, shifted in $\mathrm{CO}_{2}$ gas, was frequency doubled and used for high resolution molecular spectroscopy in a supersonic beam. For demonstration the $6_{1}^{0}$ hot band of benzene molecules is shown in fig. 5. It was measured with the technique of mass selected two-photon ionization via a resonant intermediate state [23]. Ionization was achieved by a second delayed frequency-doubled pulsed dye laser. For the experimental resolution of $160 \mathrm{MHz}$ (see insert in fig. 5) most of the rotational lines in the vibronic $6_{1}^{\circ}$ transition are resolved.

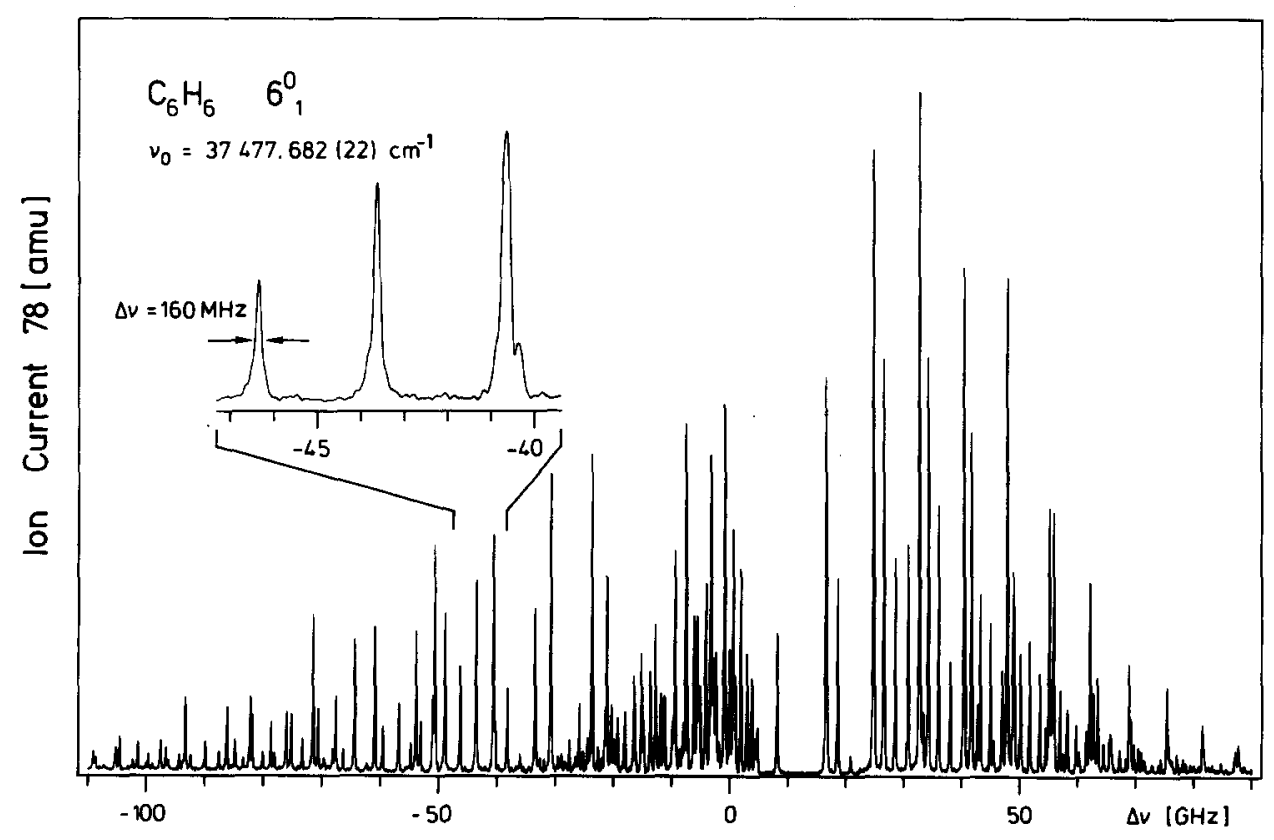

Fig. 5. Rotationally resolved spectrum of the $6_{1}^{0}$ hot band of benzene $\mathrm{C}_{6} \mathrm{H}_{6}$ measured with the narrow-band Raman shifted Stokes light. For demonstration of the experimental linewidth a portion of the spectrum is shown on an expanded scale. For details, see text. 
The high resolution and the good signal-to-noise ratio in the spectrum enabled us to determine the rotational constants $B_{6}^{\prime \prime}, C_{6}^{\prime \prime}, B_{0}^{\prime}$ and $C_{0}^{\prime}$, the Coriolis coupling constant $\zeta_{6}^{\prime \prime}$ and the band origin $\nu_{0}$ with high precision on the basis of a symmetric top model [19]. A detailed description of this analysis will be given elsewhere, here only the results will be summarized briefly.

In a first step the ground state constant $B_{6}^{\prime \prime}$ was fitted to combination differences of lines with constant $J^{\prime}, K^{\prime}$, and $\Delta K$ but differing $\Delta J$. Next the constant $C_{6}^{\prime \prime}$ was calculated from the theoretically known rotational defect $\Delta=h / 8 \pi^{2} c(1 / C-2 / B)=0.0570 \mathrm{amu}$ $\AA^{2}$ for the $6_{1}$ state [24]. Lastly, a fit of the remaining constants $\zeta_{6}^{\prime \prime}, B_{0}^{\prime}$, and $C_{0}^{\prime}$ to 81 unblended lines was performed, yielding a standard deviation of 22.3 MHz. The results are

$B_{0}^{\prime}=0.181721 \mathrm{~cm}^{-1}, \quad C_{0}^{\prime}=0.090889 \mathrm{~cm}^{-1}$,

$B_{6}^{\prime \prime}=0.189721 \mathrm{~cm}^{-1}, \quad C_{6}^{\prime \prime}=0.094830 \mathrm{~cm}^{-1}$,

$\zeta_{6}^{\prime \prime}=0.5829, \quad \nu_{0}=37477.68(2) \mathrm{cm}^{-1}$.

The values of $B_{0}^{\prime}$ and $C_{0}^{\prime}$ represent the first direct determination of the rotational constants of the zero point $S_{1}$ state in benzene. They agree quite well with the values determined from the complex analysis of combination states in the $S_{1}$ state reported recently [25].

\section{Acknowledgements}

The authors are indebted to Professor E.W. Schlag for his continuous interest in the progress of this work. Financial support from the Deutsche Forschungsgemeinschaft and the Fonds der Chemischen Industrie is gratefully acknowledged.

\section{References}

[1] J.C. White, Topics in Applied Physics, Vol. 59. Stimulated Raman scattering (Springer, Berlin, 1987) p. 115.

[2] R. Saint-Loup, B. Lavorel, G. Millot, C. Wenger and H.C. Berger, J. Ram. Spectr. 21 (1990) 77.

[3] R.H. Dicke, Phys. Rev. 89 (1953) 472.

[4] P. Lallemand, P. Simova and G. Bret, Phys. Rev. Lett. 17 (1966) 1239.

[5] J.R. Murray and A. Javan, J. Mol. Spectrosc. 29 (1969) 502.

[6] J. Kleinschmidt, H.G. Walther and B. Wilhelmi, Wiss. Ztschr. Friedrich-Schiller-Univ. Jena, Math.-Nath. R. 22 (1973) 287.

[7] F. De Martini, F. Simoni and E. Santamato, Optics Comm. 9 (1973) 176.

[8] A. Owyoung, Optics Lett. 2 (1978) 91.

[9] V.I. Alekseev and I.I. Sobel'man, Sov. Phys. JETP 28 (1969) 991.

[10] R. Roy, D.S. Elliot, D. Meschede, F.M. Pipkin and S.M. Smith, Chem. Phys. Lett. 93 (1982) 603.

[11] B. Lavorel, G. Millot, R. Saint-Loup and H. Berger, J. Chem. Phys. 93 (1990) 2185.

[12] V. Wilke and W. Schmidt, Appl. Phys. 18 (1979) 177.

[13] R. Trutna and R.L. Byer, Appl. Optics 19 (1980) 301.

[14] H. Kogelnik and T. Li, Appl. Optics 5 (1966) 1550.

[15] D. Herriot, H. Kogelnik and R. Kompfner, Appl. Optics 3 (1964) 523.

[16] A. Owyoung, Ch.W. Patterson and R.S. McDowell, Chem. Phys. Lett. 59 (1978) 156.

[17] A.E. Siegman, Lasers (Oxford Univ. Press, Oxford, 1986).

[18] E. Riedle, R. Moder and H.J. Neusser, Optics Comm. 43 (1982) 388.

[19] E. Riedle, Th. Knittel, Th. Weber and H.J. Neusser, J. Chem. Phys. 91 (1989) 4555.

[20] B. Lavorel, private communication.

[21] V.A. Chirkov, V.S. Gorelik, G.V. Peregudov and M.M. Sushchinskii, ZhETF Pis. 10 (1969) 416.

[22] S. Gerstenkorn and P. Luc, Atlas du spectre d'absorption de la molecule de l'iode (CNRS, Paris, 1978).

[23] Th. Weber, A. von Bargen, E. Riedle and H.J. Neusser, J. Chem. Phys. 92 (1990) 90.

[24] M.F. Jagod and T. Oka, J. Mol. Spectrosc. 139 (1990) 313.

[25] E. Riedle and J. Pliva, Chem. Phys. 152 (1991) 375. 